Article

\title{
Strong Effect of Hydrodynamic Coupling on the Electric Dichroism of Bent Rods
}

Dietmar Porschke, and Jan M. Antosiewicz

J. Phys. Chem. B, 2005, 109 (2), 1034-1038• DOI: 10.1021/jp046009v • Publication Date (Web): 24 December 2004

Downloaded from http://pubs.acs.org on March 23, 2009

\section{More About This Article}

Additional resources and features associated with this article are available within the HTML version:

- $\quad$ Supporting Information

- $\quad$ Links to the 1 articles that cite this article, as of the time of this article download

- $\quad$ Access to high resolution figures

- $\quad$ Links to articles and content related to this article

- Copyright permission to reproduce figures and/or text from this article

View the Full Text HTML 


\title{
Strong Effect of Hydrodynamic Coupling on the Electric Dichroism of Bent Rods
}

\author{
Dietmar Porschke*, ${ }^{*}$ and Jan M. Antosiewicz \\ Max Planck Institut für Biophysikalische Chemie, 37077 Göttingen, Germany, and Department of Biophysics, \\ Warsaw University, 02-89 Warsaw, Poland
}

Received: September 4, 2004; In Final Form: November 2, 2004

\begin{abstract}
The effect of hydrodynamic coupling on the spatial orientation of rigid bent rods in electric fields has been analyzed by Brownian dynamics simulations. Bead models for smoothly bent rods were constructed with dimensions of DNA double helices, and established simulation procedures were used to calculate their diffusion tensor, including the translational-rotational coupling tensor. The electric and optical parameters were assigned on the basis of known properties of double helices. Brownian dynamics simulations of the orientation of these models in electric fields showed that both transients and amplitudes of the calculated dichroism are very strongly dependent on translational-rotational coupling over a wide range of electric field strengths. For example, the stationary dichroism of a smoothly bent $179 \mathrm{bp}$ DNA fragment calculated at low field strengths is positive in the presence and negative in the absence of hydrodynamic coupling. The transients are converted from a biphasic to a monophasic shape, when hydrodynamic coupling is turned off. The large changes resulting from hydrodynamic coupling were controlled by calculations based on analytical expressions derived for electrooptical response curves in the limit of low electric field strengths; the results obtained by this independent approach are in very satisfactory agreement with our Brownian dynamics simulations. The effect is strongly dependent on the electric dipole and on its direction. In the absence of any dipole the coupling effect was not observed. The coupling effect increases with the size of the bent rods. Because most macromolecular structures are known to have induced and/or permanent dipole moments, large effects of hydrodynamic coupling on both the amplitudes and the transients of the electric dichroism/birefringence must be expected in general for structures with nonsymmetric shape.
\end{abstract}

\section{Introduction}

Electrooptical techniques are known to be very useful for a particularly sensitive analysis of structures and dynamics of polymers and colloids in solution. ${ }^{1-3}$ The interpretation of experimental data obtained by measurements of the electric dichroism, electric birefringence, and related parameters is simple, provided that the objects are symmetric like spheres or straight rods. Deviations from symmetry may cause special contributions resulting from hydrodynamic interactions. Although the existence of these hydrodynamic interactions has been described by Brenner ${ }^{4,5}$ already in 1965 as coupling between translational and rotational motion, contributions from this coupling effect have been ignored in most cases. This is mainly due to the difficulty of their quantitative description, which requires extensive calculations. Wegener ${ }^{6}$ derived expressions for the transient electric birefringence in the Kerr limit of low field strengths for rigid bodies of arbitrary shape. Some electrooptical data have been calculated by Brownian dynamics with consideration of hydrodynamic coupling. Antosiewicz et al. ${ }^{7}$ analyzed the case of tRNA and found an influence of hydrodynamic coupling that was clearly noticeable but remained a relatively small contribution. Recently Bertolotto et al.,9 concluded that hydrodynamic coupling causes a positive dichroism component for bent DNA double helices. This is consistent with a positive dichroism component contributed by hydrodynamic coupling in the case of tRNA, ${ }^{7}$ which may be regarded as a special case of a bent rod.

* Corresponding author.

Max Planck Institut für Biophysikalische Chemie

$\doteqdot$ Warsaw University.
The coupling effect between translational and rotational diffusion discussed in the present investigation is expected to be reflected in simulations on flexible bead assemblies, when hydrodynamic interaction effects between individual beads are included. The effect of electric fields on such flexible bead assemblies has been analyzed in several investigations. ${ }^{10-13}$ According to Diaz et al. ${ }^{11}$ hydrodynamic interactions affect the transients but not the stationary degree of orientation. Elvingson ${ }^{12}$ concluded that electric fields induce a deformation of flexible chains into bent conformations due to hydrodynamic coupling.

The present investigation is restricted to rigid models and the term "hydrodynamic coupling" refers to the coupling between translational and rotational diffusion as defined by Brenner. ${ }^{4,5}$ The coupling effect has been analyzed by Brownian dynamics simulations with particular emphasis on models for DNA double helices. The results of these simulations clearly show a huge effect of hydrodynamic coupling on electrooptical transients and demonstrate a strong dependence of this effect on the electric parameters.

Algorithm Used for Brownian Dynamics Simulations. The simulation of rotational diffusion of macromolecules in solution is based on the Brownian dynamics algorithm described by Ermak and McCammon, ${ }^{14}$ and was described ${ }^{7,15}$ in detail previously; therefore, only essential aspects of the simulation procedure are presented here.

Our program calculates temporal changes of the reduced linear dichroism $\xi$ of a "solution" of $N$ independent particles under the influence of a rectangular electric field pulse of strength $E$. The reduced dichroism is defined as

$$
\xi=\left(A_{\|}-A_{\perp}\right) / \bar{A}
$$


where $A_{\|}$and $A_{\perp}$ designate the absorbance of light polarized parallel and perpendicular, respectively, to the direction of the external electric field, and $\bar{A}$ is the average absorption of the solution. The direction of the field defines the $z$-axis of the laboratory coordinate system (LCS). Initially the particles are distributed uniformly among $8 \pi^{2}$ orientational states. Then, an electric field $E$ is applied for a time interval $t_{\text {pulse, }}$ and the trajectories of the rotational motion for each particle are calculated during some time $t_{\mathrm{obs}}$.

We define the particle coordinate system (PCS) corresponding to the system of principal axes of its rotational diffusion tensor $\hat{D}_{\mathrm{t}}$, and centered at the particle center of diffusion (CD). ${ }^{5,16}$ All relevant properties of the investigated particle, i.e., its permanent dipole moment vector $\vec{\mu}_{\mathrm{CD}}$, electric polarizability tensor, $\hat{\alpha}$, extinction coefficient tensor, $\hat{\epsilon}$, rotational diffusion tensor, $\hat{D}_{\mathrm{r}}$, translational diffusion tensor, $\hat{D}_{\mathrm{t}, \mathrm{CD}}$, and translation-rotation coupling diffusion tensor, $\hat{D}_{\mathrm{c}, \mathrm{CD}}$, are given in this coordinate system. The subscript $\mathrm{CD}$ indicates quantities that depend on the location of the origin of PCS within the particle.

The orientation of the LCS with respect to the PCS is described using the rotation vector formalism. ${ }^{17,18}$ The rotation vector $\vec{k}$ is defined by the direction of the rotation axis and the rotation angle $\chi$ for the transformation from the LCS to the PCS. The direction of the rotation axis is given by the unit vector, $\vec{u}$ $=\vec{k} / k$, where $k$ is the length of the vector $\vec{k}$ corresponding to the tangens of half of the rotation angle $\chi$. If $\vec{k}_{0}$ is the rotation vector that describes the orientation of the LCS with respect to the PCS at "zero" time, and $\vec{k}_{\text {step }}$ describes the rotation of the particle during time step $\Delta t$, then the new orientation is given by

$$
\vec{k}=\left(1-\vec{k}_{0} \cdot \vec{k}_{\text {step }}\right)^{-1}\left(\vec{k}_{0}+\vec{k}_{\text {step }}+\vec{k}_{0} \times \vec{k}_{\text {step }}\right)
$$

where $\cdot$ and $\times$ refer to scalar and vectorial products of vectors, respectively. The $\vec{k}_{\text {step }}$ is computed as

$$
\vec{k}_{\text {step }}=\frac{\hat{D}_{\mathrm{r}} \cdot \vec{T}_{0, \mathrm{CD}} \Delta t}{k T}+\frac{q \hat{D}_{\mathrm{c}, \mathrm{CD}} \cdot \vec{E}_{0} \Delta t}{k T}+\vec{K}_{\mathrm{rnd}}(\Delta t)
$$

where quantities with subscript $o$ are taken at the beginning of time step $\Delta t$.

$$
\hat{T}_{0, \mathrm{CD}}=-\left(\vec{\mu}_{\mathrm{CD}}+\hat{\alpha} \cdot \vec{E}_{0}\right) \times \vec{E}_{0}
$$

is the torque due to the electrical anisotropy of the particle with respect to the CD. $\vec{E}_{0}$ is the external electric field vector as "seen" in the PCS, $q$ is the total electric charge of the particle, $k T$ is the product of the Boltzmann constant $k$ and the absolute temperature $T$, and $\vec{K}_{\text {rnd }}(\Delta t)$ designate three random rotational steps taken as the three last elements of the generalized, 6-element, translational-rotational random step $\vec{S}$ with zero mean and the standard deviation determined by its variance-covariance matrix

$$
\left\langle S_{i} S_{j}\right\rangle=2\left(\begin{array}{ll}
\hat{D}_{\mathrm{t}, \mathrm{CD}} & \hat{D}_{\mathrm{c}, \mathrm{CD}}^{\dagger} \\
\hat{D}_{\mathrm{c}, \mathrm{CD}} & \hat{D}_{\mathrm{r}}
\end{array}\right) \Delta t
$$

with $i, j=1,2, \ldots, 6,\langle\cdot\rangle$ meaning average over the ensemble, and $\hat{D}_{\mathrm{c}, \mathrm{CD}}^{\dagger}$ being the transpose of $\hat{D}_{\mathrm{c}, \mathrm{CD}}$. When the external electric field pulse is terminated, each $\vec{k}_{\text {step }}$ is determined only by components of the rotational random step.

In the presence of a homogeneous external electric field, the absorbance of the solution shows cylindrical symmetry around the field vector, and it can be written that

$$
A_{\|} \sim\left\langle\hat{\epsilon}_{z z}^{\mathrm{LCS}}\right\rangle \text { and } A_{\perp} \sim \frac{\left\langle\hat{\epsilon}_{x x}^{\mathrm{LCS}}+\hat{\epsilon}_{y y}^{\mathrm{LCS}}\right\rangle}{2}
$$

where $\hat{\epsilon}_{i i}^{\mathrm{LCS}}$ is the $i i$ component of the $\hat{\epsilon}$ tensor in the LCS. Therefore, the reduced dichroism can be represented as

$$
\xi=\frac{3\left\langle\hat{\epsilon}_{z z}^{L C S}\right\rangle-\operatorname{Tr} \hat{\epsilon}}{2 \operatorname{Tr} \hat{\epsilon}}
$$

where $T r \hat{\epsilon}$ is the sum of diagonal elements of the tensor, and the $z z$ component of the absorbtivity tensor $\epsilon$ in the LCS is computed as

$$
\hat{\epsilon}_{z z}^{\mathrm{LCS}}=\vec{e}^{\dagger} \cdot \hat{\epsilon} \cdot \vec{e}
$$

where $\vec{e}$ is the unit vector along the $z$-axis of the LCS as "seen" in the PCS. Therefore it is sufficient to know the orientation of the $z$-axis of the LCS, which coincides with the direction of the external electric field, when the pulse is on, to compute the resulting dichroism. The rotational Brownian dynamics algorithm described above provides us with the components of the LCS $z$-axis in the PCS of each particle at each moment of time, and these components are used to compute the contribution to the reduced dichroism due to each particle. The reduced linear dichroism of the solution is calculated as the average of all individual contributions.

Construction of Models. A simple model for bent forms of DNA double helices is the smoothly bent rod. We have used this model, which is a reasonable approximation for relatively low chain lengths in the range close to the persistence length, for our present simulations. The optical and electrical parameters of this model have been discussed previously. ${ }^{19}$

One of our smoothly bent rods has been constructed to represent a DNA with $179 \mathrm{bp}$. Based on a persistence length of $500 \AA,^{20}$ the root of mean square end-to-end distance is $\sim 507$ $\AA$, which represents an average bending angle of $\sim 116^{\circ}$. A bead model representing such a bent rod with 101 beads (radius 12.5 $\AA$ ) is shown in Figure 1. This bead model is introduced into our algorithm for the calculation of hydrodynamic and electrooptical parameters. ${ }^{21}$ The diffusion tensor (eq 9) has been calculated for the coordinate system originating at the center of diffusion. The $3 \times 3$ tensor in the upper left corner represents the tensor for translational diffusion $\left[\mathrm{m}^{2} \mathrm{~s}^{-1}\right]$, whereas the $3 \times$ 3 tensor for rotational diffusion $\left[\mathrm{s}^{-1}\right]$ is represented in the lower right corner. The $3 \times 3$ tensors in the upper right and lower left corners are the tensors for translational-rotational coupling $\left[\mathrm{m} \mathrm{s}^{-1}\right]$ and its transpose, respectively. Some of the elements in the translational-rotational coupling tensor are clearly nonzero. For symmetric bead models, all the elements of the translationalrotational coupling tensor are zero. Because the magnitude of the tensor components is essential for the simulation, we have controlled our results by a simulation with the same bead model but using the algorithm of Garcia de la Torre, ${ }^{22}$ which is based on the same basic principles but has been assembled independently. The coefficients for translational diffusion were equivalent within $0.2 \%$, the two lower coefficients for rotational diffusion within 4 to $5 \%$ and the coupling coefficients within $8 \%$. The difference is due to the special form of volume correction $^{21}$ used in our bead model simulations.

The optical and electrical parameters were determined as described by Antosiewicz and Porschke. ${ }^{19}$ The coefficients of the diagonal elements of the absorbance tensor for the model shown in Figure 1 are 0.35507, 0.4665, and 0.17842 (normalized to trace $=1$; the absolute magnitude does not matter, because 


$$
\hat{D}_{\mathrm{CD}}=\left(\begin{array}{llllll}
.236 E-10 & .000 E+00 & .000 E+00 & .000 E+00 & .000 E+00 & .000 E+00 \\
.000 E+00 & .218 E-10 & .000 E+00 & .000 E+00 & .000 E+00 & .198 E-03 \\
.000 E+00 & .000 E+00 & .300 E-10 & .000 E+00 & .198 E-03 & .000 E+00 \\
.000 E+00 & .000 E+00 & .000 E+00 & .556 E+05 & .000 E+00 & .000 E+00 \\
.000 E+00 & .000 E+00 & .198 E-03 & .000 E+00 & .594 E+05 & .000 E+00 \\
.000 E+00 & .198 E-03 & .000 E+00 & .000 E+00 & .000 E+00 & .493 E+06
\end{array}\right)
$$

we always calculate the reduced dichroism). The diagonal elements of the polarizability tensor were based on the experimental values in the range of $3 \times 10^{-32} \mathrm{Cm}^{2} \mathrm{~V}^{-1}$; the ratio of the $x$ - and the $z$-components $(0.27893$ and 0.72107 , respectively) was approximated as described by Antosiewicz and Porschke. ${ }^{19}$ The net charge was calculated from the total number of phosphates and the relative charge $0.12 \times \mathrm{e}$ per phosphate resulting from polyelectrolyte theory for the case of condensation of bivalent ions. ${ }^{23}$ The permanent dipole with respect to the center of diffusion $\mu=8.96 \times 10^{-27}[\mathrm{Cm}]$ was calculated as discussed by Antosiewicz and Porschke. ${ }^{24}$

\section{Results}

Hydrodynamic Coupling Effects and Controls. Our simulations started from a state with random orientation of our models. We applied an electric field pulse at time 0 and calculated the resulting dichroism as a function of time up to a stationary level. Then the electric field pulse was turned off and the back-relaxation to the initial state was followed. Examples for the case of the smoothly bent rod described above are given in Figure 2 for a relatively low electric field strength, where a large number of transients $\left(1.1 \times 10^{6}\right)$ had to be averaged for a reasonable signal-to-noise ratio. The transients were calculated in the presence of hydrodynamic coupling by using the full diffusion tensor given above. The transients in the absence of hydrodynamic coupling were simulated by the same procedure, but all elements of the coupling tensor were turned to zero. The example in Figure 2 shows a huge effect of hydrodynamic coupling: the stationary value of the dichroism changes its sign, and the shape of the transients is quite different. The field induced orientation in the absence of hydrodynamic coupling has also been simulated with all elements of the tensor including those describing coupling but by turning the net charge to zero: the result was equivalent within the limits of simulation accuracy.

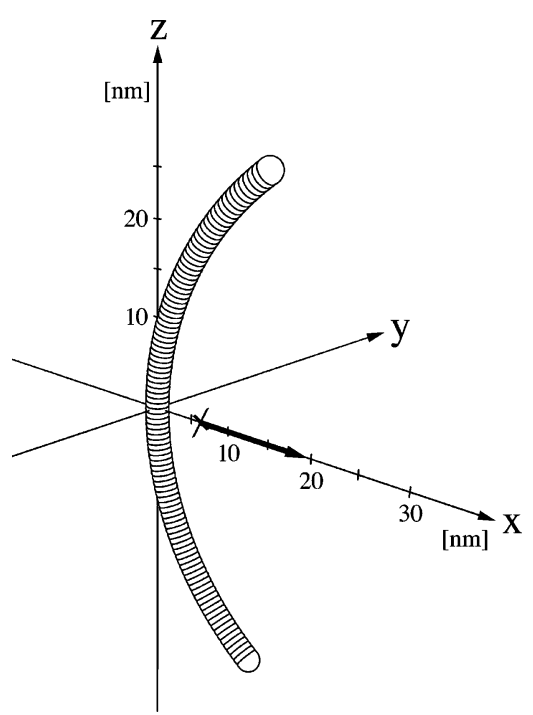

Figure 1. Model of the smoothly bent 179 bp DNA with 101 beads of $12.5 \AA$ diameter. Bending angle $116^{\circ}$. The position of the center of diffusion is indicated by the $\times$-sign. The direction of the permanent dipole moment is indicated by the arrow.
The validity of the Brownian dynamics algorithm was checked by comparison of results obtained for simple limit cases, such as time constants for objects of simple shape and stationary dichroism for simple permanent/induced dipoles. The results obtained for all these tests were in quantitative agreement with predictions by analytical theory. A test for the validity of the algorithm for hydrodynamic coupling required a special approach. Wegener ${ }^{6}$ presented equations for electrooptical transients by an analytical procedure, valid in the limit of low electric field strengths. His equations were used for independent calculations. The results shown in Figure 2 are in satisfactory agreement with those obtained by Brownian dynamics and, thus, support the validity of the Brownian dynamics algorithm.

The approach of Wegener ${ }^{6}$ is limited to low field strengths, whereas the Brownian dynamics simulations can be extended to any level of electric field strengths. The effect of hydrodynamic coupling has been simulated for the smoothly bent 179 bp DNA over a wide range of electric field strengths (Figures 3 and 4). A comparison of the transients with and without the coupling effect shows that the difference is reduced at high electric field strengths. This is due to the strong increase of the torque resulting from the induced dipole with the square of the field strength. Thus, at high field strengths the molecular orientation is dominated by the induced dipole, and the influence of other contributions including hydrodynamic coupling is negligible.

Factors Contributing to the Magnitude of Hydrodynamic Coupling. The results described above for the smoothly bent rod demonstrate that hydrodynamic coupling contributes a positive dichroism component, corresponding to a preferential orientation of the bent rod with its arc in perpendicular direction

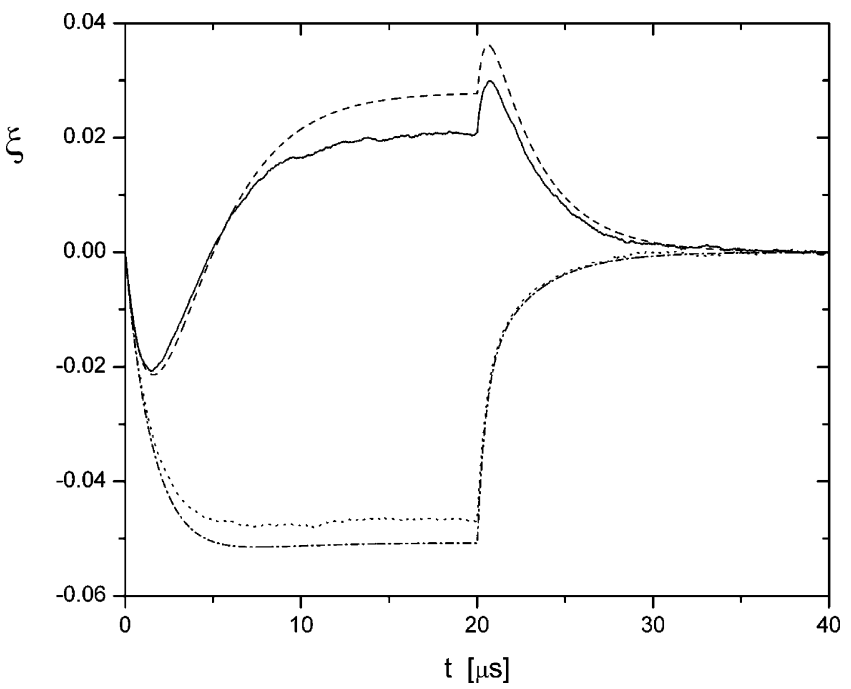

Figure 2. Reduced electric dichroism $\xi$ as a function of time $t$ calculated for the smoothly bent $179 \mathrm{bp}$ DNA (cf. Figure 1). The electric field pulse of $5 \mathrm{kV} / \mathrm{cm}$ was applied at time zero and terminated after $20 \mu$ s. The transients obtained by Brownian dynamics were calculated with and without hydrodynamic coupling ( - and $\cdots \cdot$, respectively; average of $1.1 \times 10^{6}$ individual transients). The transients were also calculated by the equations of Wegener for the limit of low electric field strength with and without hydrodynamic coupling (- - - and $-\cdot-\cdot$, respectively). 

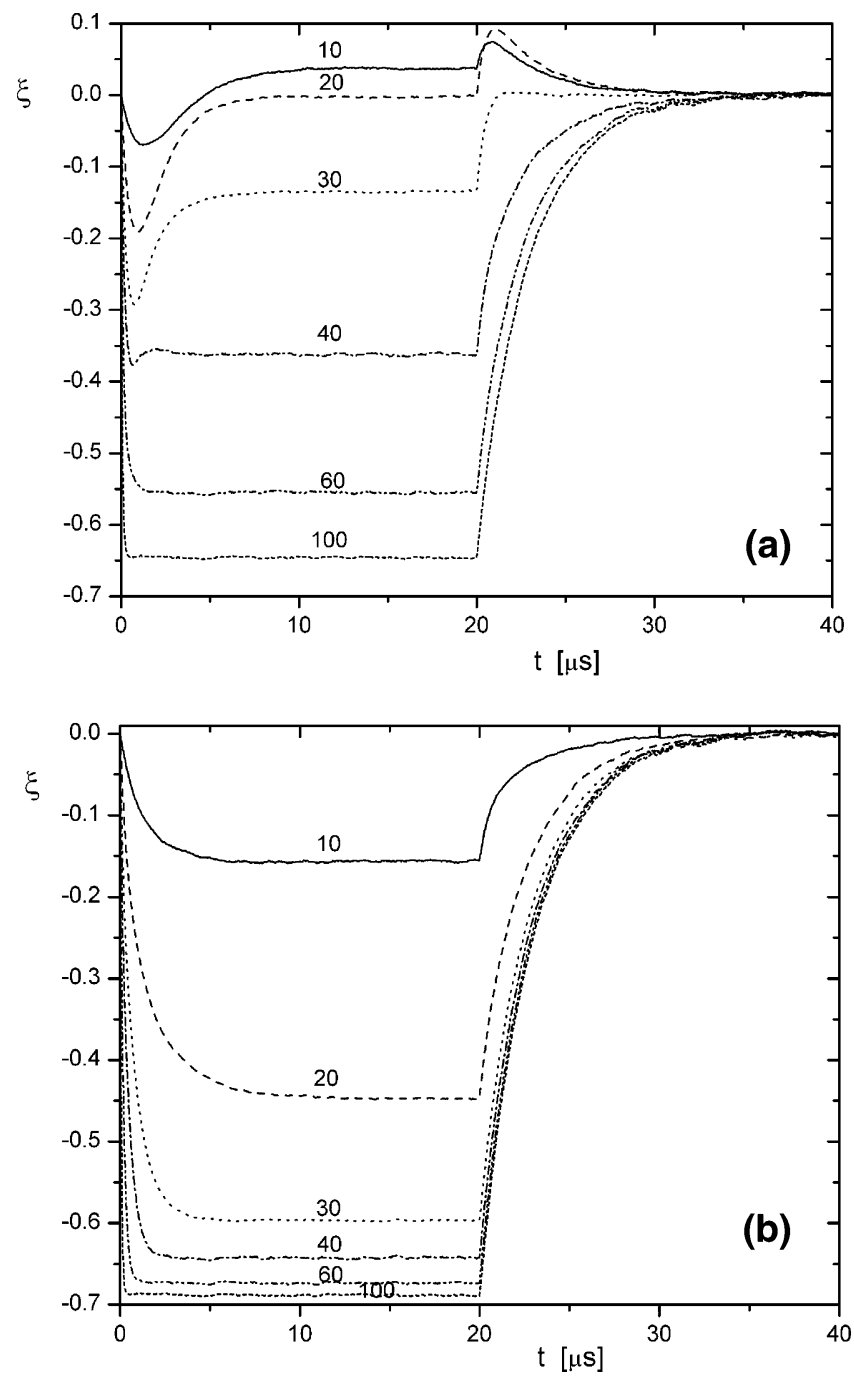

Figure 3. Reduced electric dichroism $\xi$ as a function of time $t$ for the smoothly bent 179 bp DNA calculated with (a) and without (b) hydrodynamic coupling at different electric field strengths. The field pulses were applied at $t=0$ and terminated at $t=20 \mu \mathrm{s}(E=10$ $\mathrm{kV} / \mathrm{cm}-; 20 \mathrm{kV} / \mathrm{cm}--; 30 \mathrm{kV} / \mathrm{cm} \cdots ; 40 \mathrm{kV} / \mathrm{cm} \cdot-\cdot-; 60 \mathrm{kV} / \mathrm{cm}$ $-\cdot \cdot \cdots ; 100 \mathrm{kV} / \mathrm{cm} \mathrm{-} \mathrm{-} \mathrm{-).}$

to the field vector. This conclusion is confirmed by further simulations using various dipole parameters. The data shown above were simulated with a relatively large polarizability in the $x$-direction of the particle coordinate system (cf. Figure 1). When this component is turned to zero, the magnitude of the hydrodynamic coupling effect is reduced (cf. Figure 5). A corresponding effect is observed when the magnitude of the permanent dipole is reduced. The effect of hydrodynamic coupling is also reduced, when the direction of the permanent dipole is reverted (cf. Figure 5). Finally a reduction of the positive component is also found, when the total charge of the bent rod is reduced. All these effects are as expected, at least qualitatively.

However, some of the simulation results are not in line with simple intuition. One might expect that electrophoretic motion of the smoothly bent rod alone may also lead to some degree of orientation. Our simulations did not reveal any detectable degree of orientation in the absence of any permanent or induced dipole. Thus, a minimal degree of electric anisotropy seems to be required for a detectable influence of hydrodynamic coupling.

In some cases hydrodynamic coupling leads to an unexpected form of the orientation function. An example is given in Figure 5 , showing the stationary degree of orientation as a function of the field strength for different components of the polarizability

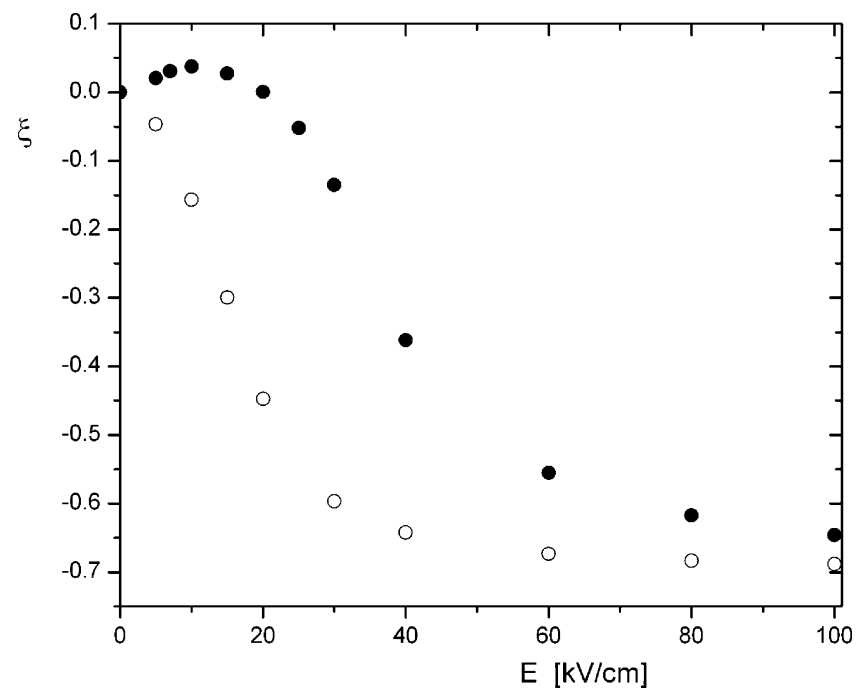

Figure 4. Stationary values of the reduced electric dichroism $\xi$ as a function of the electric field strength $E$ for the smoothly bent $179 \mathrm{bp}$ DNA in the presence $(-)$ and the absence $(O)$ of hydrodynamic coupling. The parameters of the smoothly bent rod are as described in the section "Construction of models".

in the absence of a permanent dipole. For a combination of $x$ and $z$-components of the polarizability predicted by the model of Antosiewicz and Porschke, ${ }^{19}$ the effect of hydrodynamic coupling is very small not only at high field strengths but also at low field strengths. Hydrodynamic coupling starts to be effective at intermediate values of the field strength, leading to the appearance of an additional inversion point (cf. Figure 5).

All data presented above were simulated for the same smoothly bent DNA with 179 bp. For comparison we have also simulated the electric dichroism of a smoothly bent DNA fragment with $400 \mathrm{bp}$ (Figure 6). In this case the effect of hydrodynamic coupling is higher than that found for the $179 \mathrm{bp}$ fragment. It may be expected that the effect of hydrodynamic coupling increases with the size and the asymmetry of the objects.

\section{Discussion}

Although hydrodynamic coupling has been described a long time ago by Brenner, ${ }^{4,5}$ this effect has not received sufficient attention. The present simulations demonstrate the huge influence in the case of bent rods. Doubts on the magnitude of the effect would probably remain, if the calculations would be based on a single approach only, because it is difficult to exclude errors in extensive computer programs. Fortunately, the magnitude simulated in the limit of low field strengths has been confirmed by independent calculations based on the approach of Wegener. ${ }^{6}$ Thus, the large magnitude of the effect is well documented, at least for the case of the rather long smoothly bent rods.

We have used smoothly bent rods for our calculations, because these models are a reasonable approximation for DNA double helices in the range of chain lengths close to the persistence length. The term "bent rods" has often been used in the literature for models with a sharp bend between two straight pieces. Electrooptical transients for models with local bending also show large changes induced by hydrodynamic coupling (data in preparation). The origin of the coupling effect is the loss of symmetry, whereas the details of bending do not have any strong influence.

The coupling between translational and rotational diffusion is a consequence of the more general concept of hydrodynamic interactions between parts (beads, arms, etc.) that compose a molecule. Our results illustrate the large impact of these 


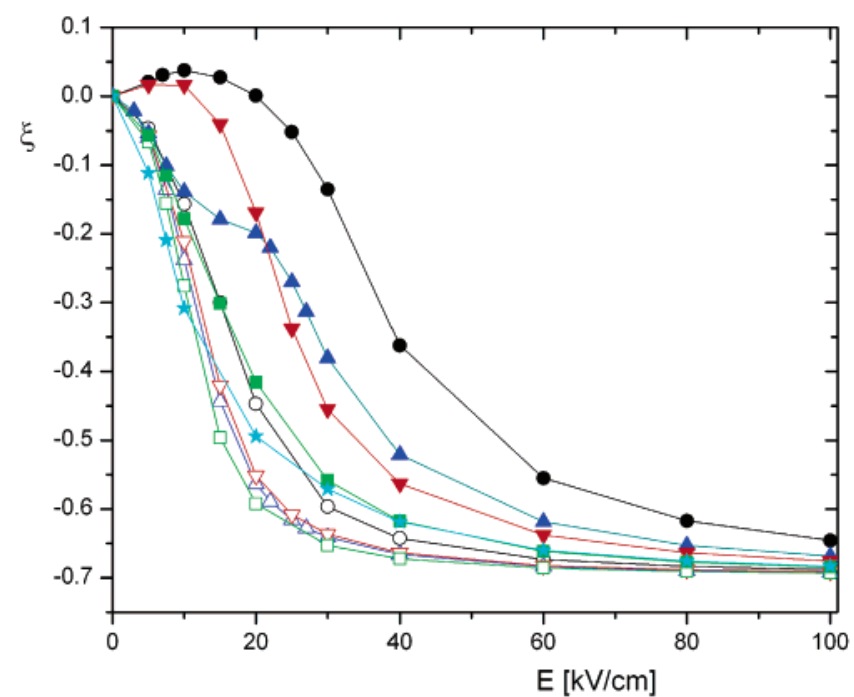

Figure 5. Stationary values of the reduced electric dichroism $\xi$ as a function of the electric field strength $E$ for the smoothly bent $179 \mathrm{bp}$ DNA, showing the magnitude of the hydrodynamic coupling effect as a function of electric parameters. Data simulated with and without hydrodynamic coupling are represented by full and empty symbols, respectively. $\bullet, \bigcirc$ (black), standard parameters of the 179 bp DNA; $\Delta, \Delta$ (blue), standard parameters, but permanent dipole turned to zero; $\boldsymbol{\nabla}, \nabla$ (red), standard parameters, but without polarizability in $\mathrm{x}$-direction; घ, $\square$ (green), standard parameters, but without permanent dipole moment and without polarizability in $x$-direction; $\star$ (cyan), standard parameters, but direction of dipole moment inverted.

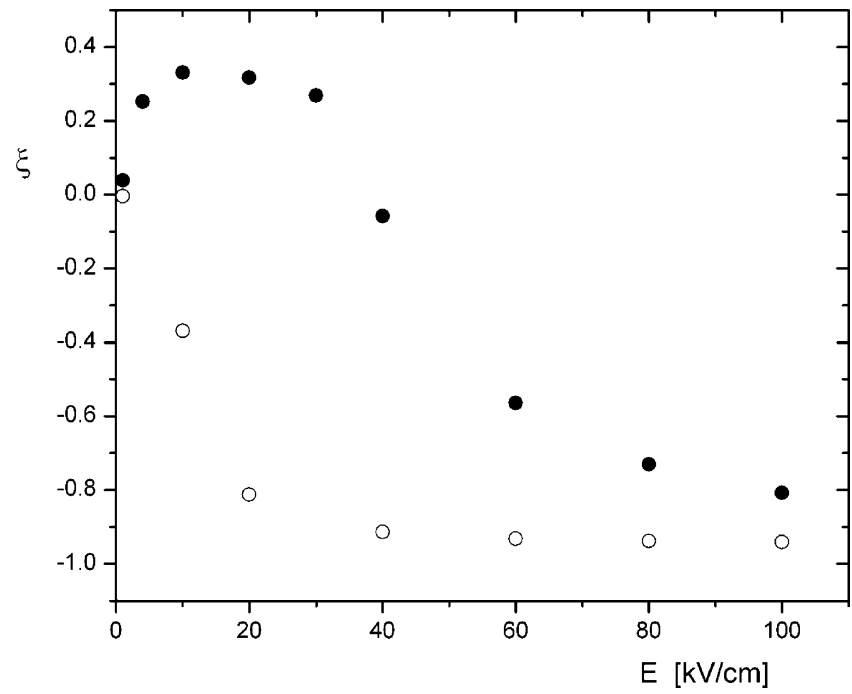

Figure 6. Stationary values of the reduced electric dichroism $\xi$ as a function of the electric field strength $E$ for a smoothly bent $400 \mathrm{bp}$ DNA in the presence $(\bullet)$ and the absence $(O)$ of hydrodynamic coupling. Bending radius $1000 \AA$; components of the extinction tensor $0.4101,0.4665,0.12131$ in $x, y$, and $z$-directions, respectively; polarizability $0.843 \times 10^{-32}$ and $5.157 \times 10^{-32}\left[\mathrm{Cm}^{2} \mathrm{~V}^{-1}\right]$ in $x$ and $z$-directions, respectively; dipole moment $1.96 \times 10^{-26}[\mathrm{Cm}]$ with respect to the center of diffusion.

hydrodynamic interactions. We have emphasized the effect of hydrodynamic coupling on stationary amplitudes. However, the simulations also show large effects on the transients. Although the overall time scale of the transients remains almost constant, the change in amplitudes of individual components induces a complete change in the overall shape of transients in many cases (cf. Figure 2). These effects are currently analyzed in more detail (data in preparation).

The hydrodynamic coupling effect is not only of mere academic interest, because experimental data for DNA fragments demonstrated a positive stationary value of the electric dichroism $^{25}$ in the range of chain lengths from 400 to $859 \mathrm{bp}$. The positive dichroism was attributed to a "permanent" dipole resulting from the anisotropy of the charge distribution of wormlike chains. The present calculations are restricted to smoothly bent rods, where the magnitude of the coupling effect is probably larger than for wormlike chains with a wide distribution of different shapes. However, on average the coupling effect is expected to provide a positive dichroism component also for the case of wormlike chains. The present simulations support the conclusion that a "permanent" dipole moment is essential for observation of a positive electric dichroism. However, the magnitude of the dipole moments required for explanation of the experimental data is not as high as discussed previously, because of the amplification effect resulting from hydrodynamic coupling.

Obviously, our present calculations are a first and elementary approach to the general problem. The structure of double helical DNA is not as simple as the smoothly bent rods used in the simulations described above. As a next step the simulations should be extended to the more irregularly shaped conformations of wormlike chains. Furthermore, the influence of the internal dynamics should be included. Simulations including all these different factors are clearly much more complex and timeconsuming.

Acknowledgment. J.M.A. was supported by Warsaw University (Grant No. BST 833/BF). We thank J.A. Bertolotto for discussions.

\section{Note Added after ASAP Publication}

This article was released ASAP on 12/24/2004. The caption for Figure 5 was modified. The revised version was posted on $12 / 30 / 2004$.

\section{References and Notes}

(1) Fredericq, E.; Houssier, C. Electric dichroism and electric birefringence; Clarendon: Oxford, 1973.

(2) Stoylov, S. P. Colloid Electrooptics; Academic Press: London, 1991.

(3) Porschke, D. In Protein-Ligand Interactions: hydrodynamics and calorimetry. A practical approach; Harding, S. E., Chowdhry, B. Z., Eds.; Oxford University Press: Oxford, 2001

(4) Brenner, H. J. Colloid Sci. 1965, 20, 104-122.

(5) Brenner, H. J. Colloid Interface Sci. 1967, 23, 407-436.

(6) Wegener, W. A. J. Chem. Phys. 1986, 84, 5989-6004.

(7) Antosiewicz, J.; Grycuk, T.; Porschke, D. J. Chem. Phys. 1991, 95, 1354-1360.

(8) Bertolotto, J.; Roston, G.; Ascheri, M.; Campo, M. Physica A 2003, $327,185-189$.

(9) Bertolotto, J. A.; Roston, G. B.; Ascheri, M. A. Prog. Colloid Polym. Sci. 2004, 128, 25-29.

(10) Navarro, S.; Lopez Martinez, M. C.; Garcia de la Torre, J. J. Chem. Phys. 1995, 103, 7631-7639.

(11) Diaz, F. G.; Carrasco, B.; Lopez Martinez, M. C.; Garcia de la Torre, J. J. Phys. Chem. 2000, 104, 12339-12346.

(12) Elvingson, C. Biophys. Chem. 1992, 43, 9-19.

(13) Elvingson, C. Chem. Phys. Lett. 1993, 214, 91-96.

(14) Ermak, D. L.; McCammon, J. A. J. Chem. Phys. 1978, 69, 13521360.

(15) Antosiewicz, J.; Porschke, D. J. Phys. Chem. 1993, 97, 2767-2773.

(16) Harvey, S. C.; Garcia de la Torre, J. Macromolecules 1980, 13, 960-964.

(17) Bialkowski, G. Mechanika Klasyczna; Polish Scientific Publisher: Warsaw, 1975.

(18) Fox, E. A. Mechanics; Harper International: New York, 1967.

(19) Antosiewicz, J.; Porschke, D. J. Biomol. Struct. Dyn. 1988, $5,819-837$

(20) Porschke, D. Biophys. Chem. 1991, 40, 169-179.

(21) Antosiewicz, J.; Porschke, D. J. Phys. Chem. 1989, 93, 5301-5305.

(22) Garcia de la Torre, J.; Navarro, S.; Lopez Martinez, M. C.; Diaz,

F. G.; Lopez Cascales, J. Biophys. J. 1994, 67, 530-531.

(23) Manning, G. Q. Rev. Biophys. 1978, 11, 179-246.

(24) Antosiewicz, J.; Porschke, D. Biophys. Chem. 1989, 33, 19-30.

(25) Porschke, D. Biophys. Chem. 1994, 49, 127-139. 